\title{
Study on the Equivalence of Penetration Overloading for Projectile-Borne Components in Nonproportional Penetrators
}

\author{
Amin Yan $\mathbb{D D}^{1,2}$ Aiguo Pi ${ }^{1},{ }^{1}$ He Yang, ${ }^{3}$ Fenglei Huang, ${ }^{1}$ and Xiaofeng Wang ${ }^{1}$ \\ ${ }^{1}$ State Key Laboratory of Explosive Science and Technology, Beijing Institute of Technology, Beijing 100081, China \\ ${ }^{2}$ State Key Laboratory of Nonlinear Mechanics, Institute of Mechanics, Chinese Academy of Sciences, Beijing 100190, China \\ ${ }^{3}$ Institute of Electronic Engineering, China Academy of Engineering Physics, Mianyang 621999, China
}

Correspondence should be addressed to Aiguo Pi; aiguo_pi@bit.edu.cn

Received 17 January 2021; Revised 13 December 2021; Accepted 16 December 2021; Published 25 February 2022

Academic Editor: Ahmed Ibrahim

Copyright (C) 2022 Amin Yan et al. This is an open access article distributed under the Creative Commons Attribution License, which permits unrestricted use, distribution, and reproduction in any medium, provided the original work is properly cited.

\begin{abstract}
The reliability assessment of the projectile-borne components in a high-speed penetrator is an important issue in the penetration field. In this study, a scaling model embedded with a deceleration measurement device was used to investigate the overloading situation due to the high cost of the prototype test. The projectile could be scaled, while the deceleration measurement device needs to maintain full scale. Thus, a nonproportional scaling design is proposed to represent the rigid-body deceleration of the prototype projectile. This study, considering the mass of the deceleration measurement device, lays out the design criteria of the scaling model and carries out rigid-body deceleration similarity verification tests of the prototype and the scaling model. In addition, the rigid-body deceleration similarity was examined through model predictions and numerical simulation. These results show that the rigid-body deceleration of the nonproportional scaling model is generally in agreement with that of the prototype for penetrating the semi-infinite concrete target. The deviations of rigid-body deceleration magnitude and duration are $6.76 \%$ and $-12.1 \%$, respectively. This makes it reasonable and feasible to investigate the overloading situation of prototype projectile through a nonproportional scaling model.
\end{abstract}

\section{Introduction}

The kinetic energy (KE) penetrators are a powerful tool used to strike hard targets such as multilayer buildings and deep underground bunkers. The fuze (or other projectile-borne components) serves as the control unit of the KE penetrator to record the acceleration data and then to control the explosion of the warhead, which needs effective protection against the shock. With the development of the KE penetrator toward high-speed and penetrating high-strength and multilayer complex media, the survivability and reliability of key components of the fuze are difficult to evaluate. Thus, a large number of experiments are required to obtain the overloading situation for the key components of fuze during the penetration event. To reduce the high cost from prototype experiments, the scaling model projectile with projectile-borne measurement device has become an important method to investigate and predict the overloading situation of prototype projectile. In the design of the scaling model, the projectile is scaled down, while the measurement device needs to remain full scale. The abovementioned issue does not fit the condition of complete similitude. Thus, we need to consider the nonproportional scaling method, so that the overloading situation of the model can accurately represent that of the prototype projectile.

In the penetration tests of the projectile, the penetration overloading is acquired by a projectile-borne measurement device. Zhang et al. [1] published numerous studies on the development of a missile-borne storage testing system under high-speed impact. They adopted the anti-high-shock device to achieve a deceleration measurement with a deceleration magnitude of more than $10^{4} \mathrm{~g}$. Glößner et al. [2] acquired the penetration overloading with a deceleration magnitude of almost $70000 \mathrm{~g}$ using an onboard data recorder system. In addition, the composition of overloading deceleration also attracts many scholars to carry out research studies $[3,4]$. It is now generally accepted that the penetration overloading consists of rigid-body deceleration, structural response of 
the penetrator and measurement sensor, and high-frequency noise. The rigid-body deceleration is a result of penetration resistance [5]. The structural response of the penetrator and measurement sensor rises from the penetration resistance transmitted from the projectile to the measuring sensor. Zhang et al. [6] investigated the force transmission mechanism of the projectile-fuze system. Based on the wavelet analysis, the frequency components of the structural response of the projectile-fuze system were explored. Guo et al. [7] studied the force transmission process based on the revised SHPB (Split Hopkinson pressure bar) and proposed that the structural response of composite structure consisted of three terms, namely, structural vibration, forced vibration, and shock response. In the abovementioned research, the rigid-body deceleration is considered as the input condition to investigate the structural response of the system. Thus, to simulate the penetration overloading inside the prototype, the equivalent rigid-body deceleration between the prototype and model should be built.

The scaling issues were developed in the past on a strong theoretical basis [8]. Based on the similarity analysis or dimensional analysis [9], numerous researchers proposed some empirical formulas to predict the terminal effect of the projectile. Anderson and Riegel [10] used the similarity theory to represent the penetration response as a function of a normalized impact velocity, which was the ratio of the penetration pressure to the target strength, and the normalized penetration depth was presented as a function of the normalized impact velocity over a very large velocity range. $\mathrm{Li}$ and Chen $[11,12]$ defined two dimensionless numbers, the impact function and the projectile geometry function, based on the similitude analysis and concrete penetration model. The penetration results can be expressed by these two dimensionless numbers for various strengths of the concrete target. Chai et al. [13] have demonstrated the similarities of rigid-body penetration by three nondimensional but physically meaningful quantities. It is shown that the experimental data of rigid projectile penetration, from shallow to deep penetration, can be uniquely unified by these three similarity quantities and their relationships. In addition, the similitude laws have also been applied in other fields, such as structural deformation [14], vibration [15, 16], and crack propagation [17]. Unfortunately, the scaling model parameters from research do not always comply with the similitude laws perfectly. When the model and the prototype cannot be associated with a single geometric scaling factor, it is referred to as incomplete similitude (nonproportional similitude) or a distorted model. The shock response from strain rate effect $[18,19]$ or geometrical distortions $[20]$ is an example.

Up to now, there have been a few works on the similarity law of rigid-body deceleration. Most of them have concentrated on a complete-similitude scaling method according to the dimensional analysis [21]. In general, once a scale factor $\lambda$ $(\lambda>1)$, the ratio of geometric dimensions, is defined, other model variables can be expressed by those of the prototype, as shown in Table 1 . To further clarify the scaling laws listed in Table 1, the deceleration-time curves of finite element analysis with different scaling factors are shown in Figure 1.
TABLE 1: Scaling factors of the small-scale model and prototype.

\begin{tabular}{lcc}
\hline Variables & Prototype & Complete-similitude model \\
\hline Length & $L$ & $L / \lambda$ \\
Time & $T$ & $T / \lambda$ \\
Mass & $M$ & $M / \lambda^{3}$ \\
Deceleration & $A$ & $\lambda A$ \\
Velocity & $V$ & $V$ \\
\hline
\end{tabular}

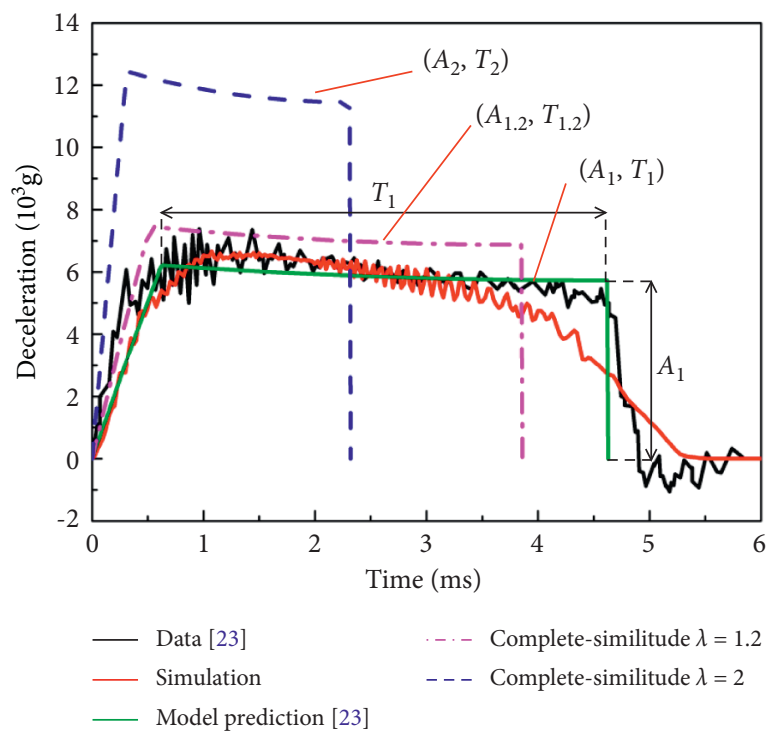

Figure 1: Deceleration-time data and model prediction with different scaling factors for projectiles penetrating the concrete target $\left(f_{c}=23 \mathrm{MPa}\right)$.

The rigid-body deceleration magnitude $A$ and duration $T$ of the complete-similitude scaling model vary with different scaling factors, and the variation amplitude depends on the scaling factor $\lambda$. To assess the penetration fuze using a scaling model, the overloading situation of the scaling model need to agree with that of the prototype. Thus, the complete-similitude scaling model cannot meet the requirement.

Moreover, since the prototype and scaling model projectile will be equipped with a deceleration measurement device of the same size and mass, the completesimilitude design cannot satisfy the structure requirements. Therefore, a nonproportional scaling method should be applied to meet the equivalence of the inner overloading situation between the prototype and the model.

Here, a design method of the nonproportional scaling model was proposed and evaluated, and the rigid-body deceleration equivalence between the prototype and the scaling model was investigated. In addition, the mass of the deceleration measurement device was considered in the design of the nonproportional scaling model, and design conditions were investigated. Finally, the reliability of the nonproportional scaling method was verified by the strategies including the penetration test, FEM, and empirical model prediction. 


\section{The Design Scheme of Nonproportional Scaling Projectile}

2.1. Representation of Geometric Scaling Factors of the Projectile. The focus of this study is a hollow ogive-nose projectile. Figure 2 shows the projectile structure. From [22], the mass expression of the projectile is given by

$$
M=\rho \frac{\pi D^{2}}{4}(l b+\beta D)-\rho \frac{\pi D^{2}(1-2 \xi)^{2}}{4} l e,
$$

where $\beta$ is the projectile head volume coefficient, which is calculated by CRH [22]. The projectile has density $\rho$, diameter $D$, shank length $l b$, and internal cavity length $l e$. The dimensionless wall thickness $\xi$ is defined as $h t / D$.

The scaling factors of mass, diameter, shank length, and internal cavity length for the prototype and model are given as follows:

$$
\begin{aligned}
& \lambda_{M}=\frac{M_{p}}{M_{m}}, \\
& \lambda_{D}=\frac{D_{p}}{D_{m}}, \\
& \lambda_{l b}=\frac{l b_{p}}{l b_{m}}, \\
& \lambda_{l e}=\frac{l e_{p}}{l e_{m}},
\end{aligned}
$$

where subscripts $p$ and $m$ refer to the prototype and the model, respectively. The scaling factors $\left(\lambda_{D}, \lambda_{l b}\right.$, and $\left.\lambda_{l e}\right)$ are not numerically identical in the design of nonproportional scaling.

2.2. Penetration Deceleration Model. From cavity-expansion analysis, Forrestal [23] described the form of rigid-body deceleration during the projectile penetration process as follows:

$$
\begin{aligned}
& a=\frac{c H}{M} 0<H<2 D, \\
& a=\frac{\pi D^{2}}{4 M}\left(R+N \rho_{t} \nu^{2}\right) H>2 D, \\
& N=\frac{8 \psi-1}{24 \psi^{2}} .
\end{aligned}
$$

Here, $H$ is the penetration depth and $v$ is the instantaneous velocity. The projectile is described by mass $M$ and $\mathrm{CRH} \psi$. Target density is $\rho_{t}$ and target resistance parameter is $R$. The calculation method is shown in [23]. For concrete $[24,25]$ and limestone [26] targets, posttest target observations showed a conical entry crater with a depth of two or more projectile diameters followed by a circular channel or tunnel. Equation (3) describes the crater phase of penetration. The constant $c$ is determined from the analysis [23],

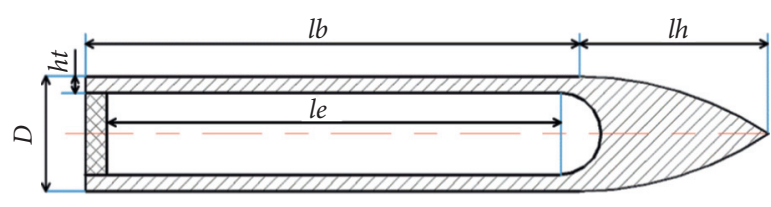

FIgURe 2: Projectile structure.

and equation (4) describes the tunnel phase. In addition, the rigid penetrator hypothesis and the semi-infinite thick target should be satisfied to obtain equation (4).

For deep penetration, we ignore the effect of the crater phase of deceleration, and only the tunnel phase is considered in the calculation. To achieve an equivalent rigidbody deceleration for the prototype and the scaling model, the following should be satisfied:

$$
\lambda_{a}=\frac{a_{p}}{a_{m}}=1,
$$

where $a_{p}$ and $a_{m}$ are the rigid-body decelerations of the prototype and scaling model, respectively. Substituting equation (4) into equation (6), the following is obtained:

$$
\frac{\lambda_{D}^{2}}{\lambda_{M}}=\frac{R+N_{m} \rho_{t} v_{m}^{2}}{R+N_{p} \rho_{t} v_{p}^{2}}
$$

The scaling model is designed to have the same $\mathrm{CRH}$, striking velocity $\nu_{o}$, and target density $\rho_{t}$ as those of the prototype. Thus, from equation (7), the relation of $\lambda_{D}$ and $\lambda_{M}$ is expressed as

$$
\lambda_{M}=\lambda_{D}^{2}
$$

2.3. Design Condition of the Nonproportional Projectile. The deceleration measurement device consists of an accelerometer, an acceleration data recorder, protective equipment, and data processing equipment. Due to the limitation of launching mass under the laboratory conditions, the mass of the deceleration measurement device cannot be ignored compared to that of the projectile, and the effect of the measurement device should be considered in the design of the scaling model. Assume that the mass ratio of the deceleration measurement device to the prototype projectile is $m_{t} / M_{p}=k$, the prototype and the scaling model adopt the deceleration measurement device of the same size and mass, and the mass scaling factor in equation (2) is revised as follows:

$$
\begin{aligned}
\lambda_{M} & =\frac{M_{p}+m_{t}}{M_{m}+m_{t}} \\
& =\frac{M_{p}+k M_{p}}{M_{m}+k M_{p}} .
\end{aligned}
$$

From equation (9),

$$
\begin{aligned}
\frac{M_{p}}{M_{m}} & =\frac{\lambda_{M}}{1+k-\lambda_{M} k} \\
& =\gamma \lambda_{M},
\end{aligned}
$$


where

$$
\gamma=\frac{1}{1+k-\lambda_{M} k}
$$

$$
\begin{aligned}
\left(\gamma \lambda_{M}\right) \cdot \rho_{m} \cdot \frac{\pi}{4} D_{m}^{2} \cdot\left(l b_{m}+\beta_{m} D_{m}\right)-\left(\gamma \lambda_{M}\right) \cdot \rho_{m} \cdot \frac{\pi}{4} D_{m}^{2}\left(1-2 \xi_{m}\right)^{2} \cdot l e_{m} & =\rho_{p} \cdot \frac{\pi}{4} D_{p}^{2} \cdot\left(l b_{p}+\beta_{p} D_{p}\right) \\
& -\rho_{p} \cdot \frac{\pi}{4} D_{p}^{2}\left(1-2 \xi_{p}\right)^{2} \cdot l e_{p} .
\end{aligned}
$$

In the design of a scaling model, the following three parameters should be the same as those of the prototype: (a) the head volume coefficient (i.e., $\beta_{m}=\beta_{p}$ ), (b) dimensionless wall thickness (i.e., $\xi_{m}=\xi_{p}$ ), and (c) projectile density (i.e., $\rho_{m}=\rho_{p}$ ). Thus, the equation (12) is simplified as follows: where

$$
\begin{aligned}
\frac{l b_{p}}{D_{m}} & =\frac{l b_{p}}{D_{m}} \cdot \frac{l b_{m}}{l b_{m}} \\
& =\frac{l b_{p}}{l b_{m}} \cdot \frac{l b_{m}}{D_{m}} \\
& =\lambda_{l b} \cdot \frac{l b_{m}}{D_{m}}, \\
\frac{l e_{p}}{D_{m}} & =\frac{l e_{p}}{D_{m}} \cdot \frac{l e_{m}}{l e_{m}} \\
& =\frac{l e_{p}}{l e_{m}} \cdot \frac{l e_{m}}{D_{m}} \\
& =\lambda_{l e} \cdot \frac{l e_{m}}{D_{m}} .
\end{aligned}
$$

Substituting equations (2), (14), and (15) into equation (13) leads to

$$
\begin{gathered}
\left(\gamma \lambda_{M}\right)\left(\frac{l b_{m}}{D_{m}}+\beta_{p}\right)-\lambda_{D}^{2} \lambda_{l b} \frac{l b_{m}}{D_{m}}-\beta_{p} \lambda_{D}^{3} \\
-\left(1-2 \xi_{p}\right)^{2} \varphi_{m}\left(\gamma \lambda_{M}-\lambda_{D}^{2} \lambda_{l e}\right)=0 .
\end{gathered}
$$

The dimensionless cavity length of the scaling model $\varphi_{m}$ is defined as $l e_{m} / D_{m} \cdot \beta_{p}$ is the head volume coefficient of the prototype projectile.

Substituting equation (8) into equation (16) leads to

$$
\beta_{p}\left(\lambda_{D}-\gamma\right)+\frac{l b_{m}}{D_{m}}\left(\lambda_{l b}-\gamma\right)=\left(1-2 \xi_{p}\right)^{2} \varphi_{m}\left(\lambda_{l e}-\gamma\right),
$$

where
Substituting equations (18) and (19) into equation (17) leads to

$$
\begin{aligned}
& \beta_{p}\left(\lambda_{D}-\gamma\right)+\frac{\lambda_{D}}{\lambda_{l b}} \cdot \frac{l b_{p}}{D_{p}}\left(\lambda_{l b}-\gamma\right) \\
= & \left(1-2 \xi_{p}\right)^{2} \cdot \varphi_{p} \frac{\lambda_{D}}{\lambda_{l e}} \cdot\left(\lambda_{l e}-\gamma\right), \\
\gamma & =\frac{1}{1+k-\lambda_{D}^{2} k} .
\end{aligned}
$$

From equation (20), when $k=0$ (or $\lambda_{D}=1$ ), it shows the design condition of the nonproportional scaling model without considering the mass of the measurement device. Figure 3 shows the flow diagram of formula derivation.

With the following prototype projectile parameters, $C H R=3, \xi_{p}=0.11, \varphi_{p}=6$, and $\left(l h_{p}+l b_{p}\right) / D_{p}=8$, the corresponding relation among $\lambda_{D}, \lambda_{l b}$, and $\lambda_{l e}$ is shown in Figure 4 , with increasing mass ratio $k$.

Figure 4 (a) shows the relation curves of $\lambda_{D}, \lambda_{l b}$, and $\lambda_{l e}$ when $k=0$, which is the case of ignoring the mass of 


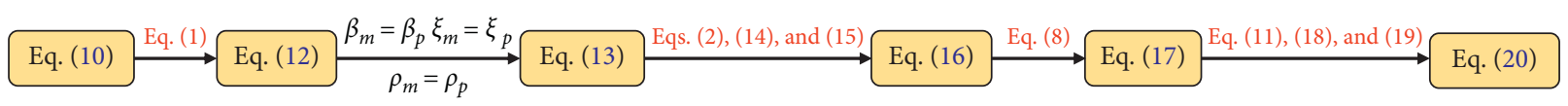

Figure 3: The flow diagram of formula derivation.
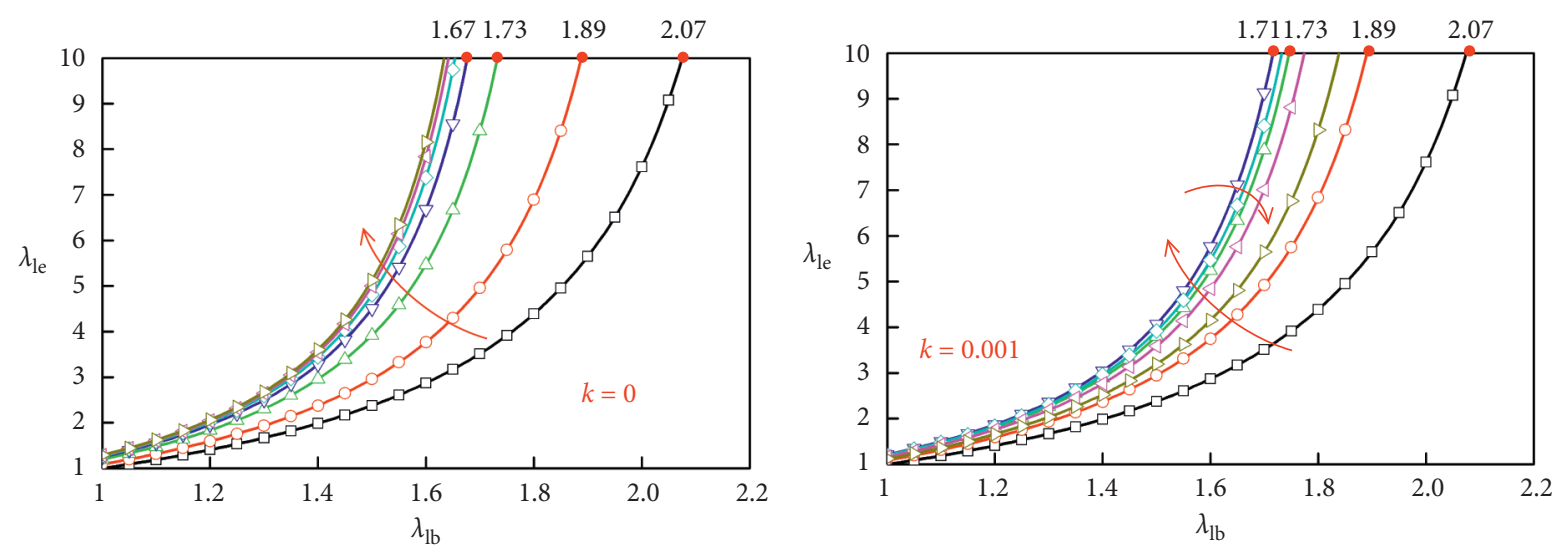

$$
\begin{aligned}
& \rightarrow-\lambda_{\mathrm{D}}=1 \\
& \multimap-\lambda_{\mathrm{D}}=1.5 \\
& \triangle \lambda_{\mathrm{D}}=3 \\
& \rightarrow-\lambda_{\mathrm{D}}=5 \\
& \checkmark \lambda_{\mathrm{D}}=7 \\
& \smile \lambda_{\mathrm{D}}=9 \\
& \rightarrow \lambda_{\mathrm{D}}=11
\end{aligned}
$$

(a)

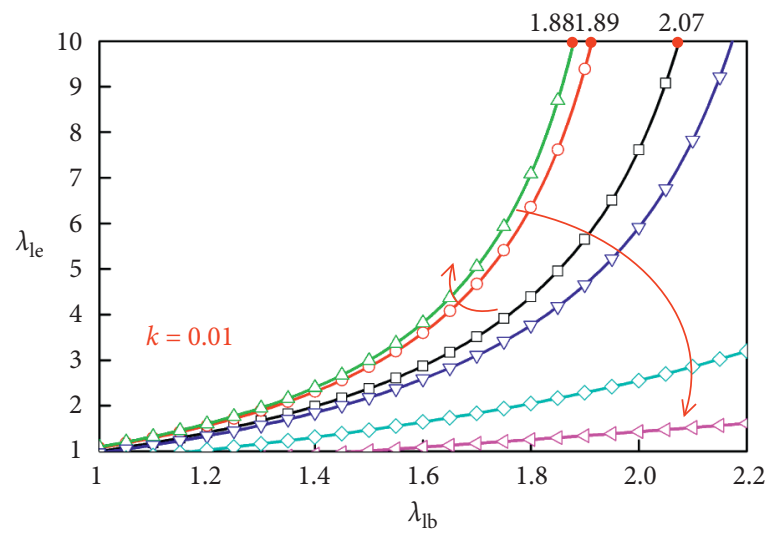

$$
\begin{array}{ll}
\neg-\lambda_{\mathrm{D}}=1 & -\lambda_{\mathrm{D}}=5 \\
\multimap-\lambda_{\mathrm{D}}=1.5 & \checkmark-\lambda_{\mathrm{D}}=7 \\
\neg-\lambda_{\mathrm{D}}=3 & \checkmark \neg \lambda_{\mathrm{D}}=9
\end{array}
$$

(c)

$$
\begin{aligned}
& \neg-\lambda_{\mathrm{D}}=1 \\
& \checkmark-\lambda_{\mathrm{D}}=7 \\
& -\circ \lambda_{\mathrm{D}}=1.5 \\
& \triangleleft-\lambda_{\mathrm{D}}=9 \\
& \triangle \lambda_{\mathrm{D}}=3 \\
& \rightarrow-\lambda_{\mathrm{D}}=5 \\
& \rightarrow \lambda_{\mathrm{D}}=11
\end{aligned}
$$

(b)

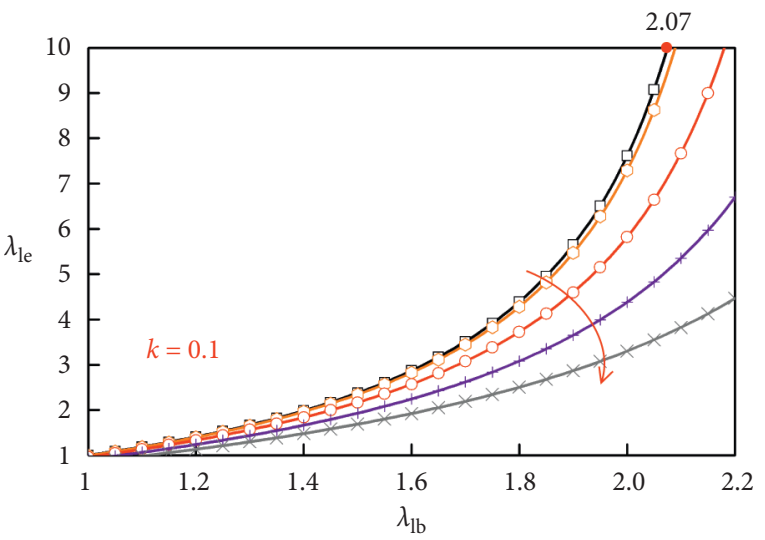

$$
\begin{array}{ll}
\multimap-\lambda_{\mathrm{D}}=1 & \longrightarrow \lambda_{\mathrm{D}}=1.75 \\
\multimap-\lambda_{\mathrm{D}}=1.25 & * \lambda_{\mathrm{D}}=2 \\
\multimap-\lambda_{\mathrm{D}}=1.5 &
\end{array}
$$

(d)

FIgure 4: Continued. 


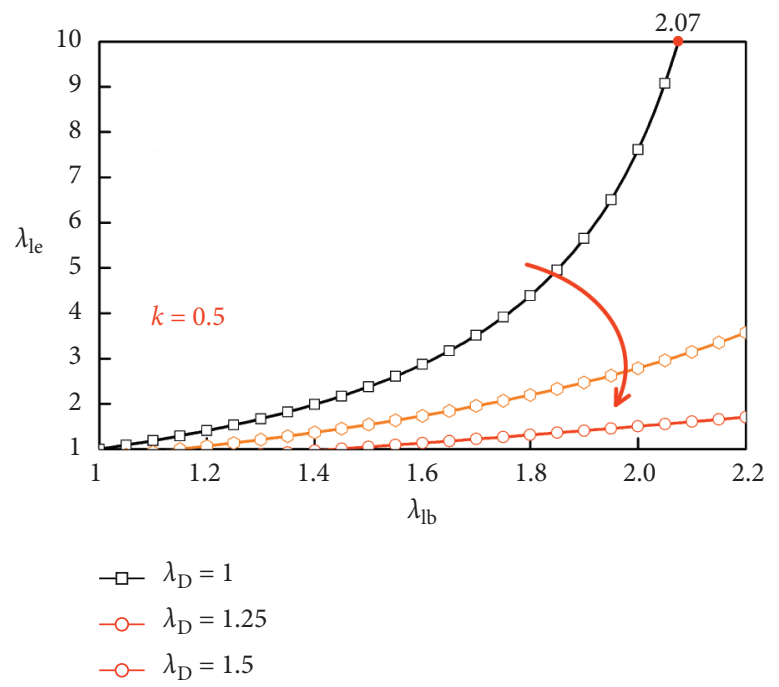

(e)

FiguRE 4: Corresponding relation of $\lambda_{D}, \lambda_{l b}$, and $\lambda_{l e}$ for the nonproportional scaling projectile: (a) $k=0$; (b) $k=0.001$; (c) $k=0.01$; (d) $k=0.1$; (e) $k=0.5$.

measurement device. When $\lambda_{D}$ is less than 3 , the curve distribution is sparse; $\lambda_{l b}$ and $\lambda_{l e}$ are sensitive to the variation of $\lambda_{D}$. However, when $\lambda_{D}$ is greater than 3 , the curve distribution become dense. The variation of $\lambda_{D}$ causes a minor change in $\lambda_{l b}$ and $\lambda_{l e}$. Figure 4(b) shows the relation curves of $\lambda_{D}, \lambda_{l b}$, and $\lambda_{l e}$ when $k=0.001$. When $\lambda_{D}$ is less than 3 , the relation curves of $\lambda_{l b}$ and $\lambda_{l e}$ are in general agreement with that in Figure 4(a). Figure $4(\mathrm{c})$ shows the relation curves of $\lambda_{D}, \lambda_{l b}$, and $\lambda_{l e}$ when $k=0.01$. When $\lambda_{D}$ is less than 1.5 , the relation curves of $\lambda_{l b}$ and $\lambda_{l e}$ are consistent with that in Figure 4(a). Thus, for a relatively small $k$ value, a critical value exists. When $\lambda_{D}$ is less than the critical value, the relation curves of $\lambda_{l b}$ and $\lambda_{l e}$ exhibit agreement with that in Figure 4(a), indicating that the mass of measurement device could be ignored. However, once $k$ increases above a certain point, the relation curves show a significant difference from those in Figure 4(a), indicating the mass of measurement device should be considered in the nonproportional scaling design (Figures $4(\mathrm{~d})$ and $4(\mathrm{e})$ ). In addition, the curves converge toward the bottom right corner as the value of $k$ increases. The value ranges of $\lambda_{D}, \lambda_{l b}$, and $\lambda_{l e}$ (especially $\lambda_{l e}$ ) become narrow. Thus, the mass of internal deceleration measurement device should be considered in the structural design of the scaling model.

\section{Equivalence Verification Tests of the Rigid- Body Deceleration}

The experiments were conducted on a $152 \mathrm{~mm}$ light-gas gun launch platform. Due to the restriction of launch caliber and mass, we designed the nonproportional projectile using a moderate factor. In this study, the mass of the deceleration measurement device and prototype projectile is $0.452 \mathrm{~kg}$ and $4.232 \mathrm{~kg}$, respectively. The mass ratio of the deceleration measurement device to the prototype projectile is 0.1068 . After considering the scaling factor relation (equation (20)), the center of mass, and the geometric dimension of the deceleration measurement device, we determined the scaling factors $\lambda_{D}, \lambda_{l b}$, and $\lambda_{l e}$ to be $1.28,1.04$, and 1.81 , respectively, as the design parameters of the scaling model.

3.1. Material and Structure of the Prototype and Model Projectile. To verify the applicability and feasibility of the nonproportional scaling method proposed in this study, a prototype and scaling model projectile were designed and machined based on the determined scaling factors. The geometric parameters and scaling factors for the prototype and the nonproportional model are listed in Table 2. The masses of the deceleration measurement device and scaling model are $0.452 \mathrm{~kg}$ and $2.4 \mathrm{~kg}$, respectively. Furthermore, as a prerequisite for obtaining the relation of scaling factors (equation (20)), the $\mathrm{CRH}$, projectile material, striking velocity, dimensionless wall thickness, and concrete target should be the same as the prototype in the design of the scaling model. The dimensionless wall thickness of the prototype and the model projectile cannot be completely consistent because of the same dimensions of the built-in deceleration measurement device. Thus, in the design of the prototype and the model, we merely guarantee that the dimensionless wall thickness is largely consistent in the installation area. Figure 5 shows the geometries of the prototype, the nonproportional scaling model, and the deceleration measurement device, respectively. To ensure the structural strength, the projectile and measurement device were machined from 30CrMnSiNi2A high-strength steel, and necessary heat treatment was performed. The deceleration measurement device was attached to the tail of the projectile by a threaded connection.

The sampling frequency and trigger threshold of the sensor were set at $120 \mathrm{kHz}$ and $8000 \mathrm{~g}$, respectively. Furthermore, a 50 min sleep time was set to prevent accidental triggers before formal sampling. As the subcaliber launch mode was used, the projectiles were fitted with sabots that 
TABLE 2: Geometric parameters and scaling factors of the prototype and scaling model.

\begin{tabular}{lccc}
\hline Variable & Prototype & Model & Scaling factor \\
\hline$D(\mathrm{~mm})$ & 64.0 & 50.0 & 1.28 \\
$l e(\mathrm{~mm})$ & 159.0 & 88.0 & 1.81 \\
$l b(\mathrm{~mm})$ & 178.9 & 172.1 & 1.04 \\
\hline
\end{tabular}

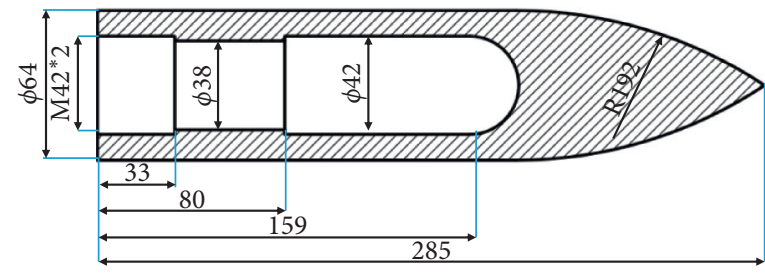

(a)

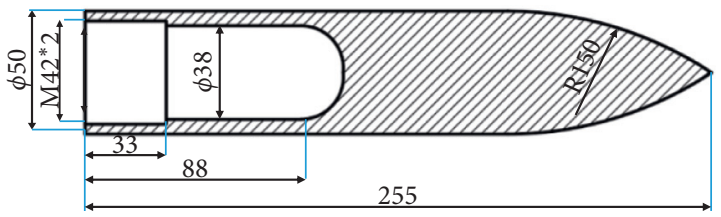

(b)

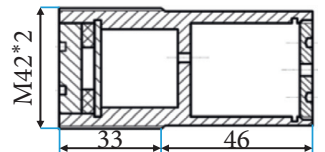

(c)

FIGURE 5: Geometries of projectiles and deceleration measurement device: (a) prototype projectile; (b) nonproportional scaling projectile; (c) deceleration measurement device (dimensions in $\mathrm{mm}$ ).

were separated from the projectiles prior to impact. The sabot was made of a lightweight and brittle material so its effects on the penetration ability could be ignored. The final launch masses of the prototype and the scaling model projectiles (including the sabot) were $7.088 \mathrm{~kg}$ and $5.286 \mathrm{~kg}$, respectively.

3.2. Testing Scheme. A $152 \mathrm{~mm}$ compressed-gas gun (in Figure 6) launched the prototype and model projectile to strike the concrete target at the same speed of $320 \mathrm{~m} / \mathrm{s}$. The projectile-target encounter condition, including the striking velocity and incident attitude angle, was measured by Photron SA5 high-speed camera, and the deceleration measurement device was adopted to capture the penetration deceleration data. The projectile penetrated the concrete vertically. The dimension of the C40 concrete target was $\varphi 1.2 \mathrm{~m} \times 0.6 \mathrm{~m}$, and the measured unconfined compression strength was $48 \mathrm{MPa}$. The diameter ratios of the target to the projectile for prototype and model are 18.75 and 24, respectively. So, the concrete target plate can be regarded as a semi-infinite target. One effective test was carried out for the prototype projectile and the scaling model projectile, respectively.

\section{Finite Element Analysis of the Prototype and Scaling Model Projectile Penetrating Semi- Infinite Concrete Target}

The penetration process of the prototype and scaling model was simulated by explicit dynamic FEM (finite element

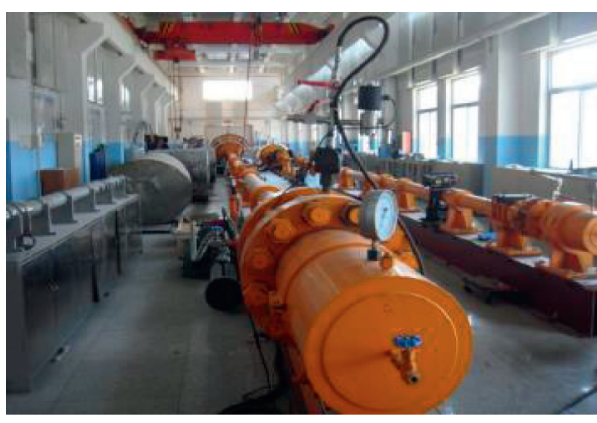

FIGURE 6: Launch platform of the $152 \mathrm{~mm}$ compressed-gas gun.

method). Figure 7 shows the finite element models of the prototype and nonproportional model (including the deceleration measurement device). Without considering the electronic parts inside the deceleration measurement device, it was simplified to a hollow cylindrical structure (in Figure 7). Because of structural symmetry, $1 / 4$ finite element model of the projectiles and measurement device were established to reduce the computational time. All entities were discretized using 8-node solid hexahedral elements with an average mesh size of $0.3 \mathrm{~cm}$. Similarly, a $1 / 4$ finite element symmetrical model of the concrete target was established based on the actual geometry. The appropriate mesh size of the interaction area of the target should be $0.5 \sim 1$ times that of the projectile nose $[27,28]$. Thus, the mesh size of the interaction area of the target was determined to be $0.2 \mathrm{~cm}$. Local mesh refinement was deployed in the mesh 


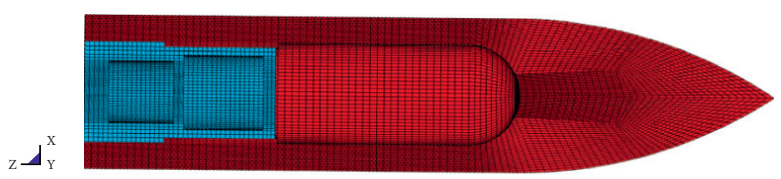

(a)

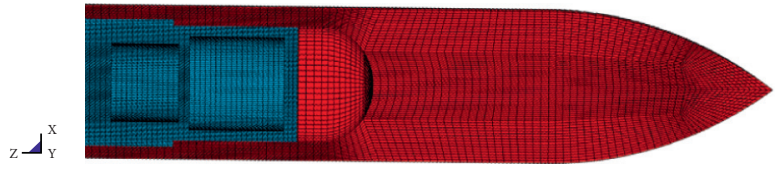

(b)

FIGURE 7: Finite element models of the projectiles (including the deceleration measurement device): (a) prototype projectile; (b) nonproportional scaling projectile.

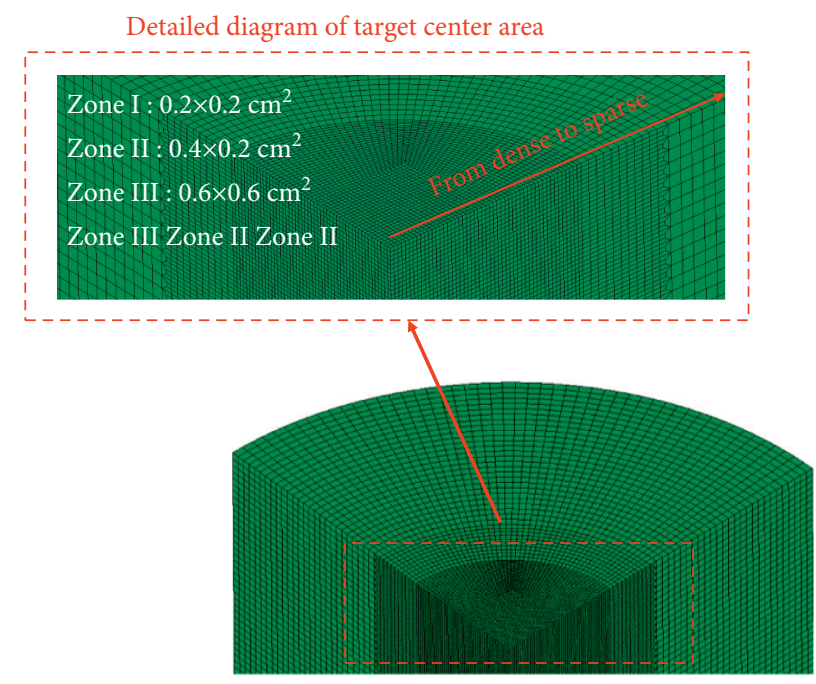

Figure 8: Finite element models of the concrete target.

division of concrete targets to guarantee the requirements of computational precision and save computational cost (in Figure 8). According to the mesh size, the concrete target plate can be divided into three areas: zone $\mathrm{I}\left(0.2 \times 0.2 \mathrm{~cm}^{2}\right)$, zone II $\left(0.4 \times 0.2 \mathrm{~cm}^{2}\right)$, and zone III $\left(0.6 \times 0.6 \mathrm{~cm}^{2}\right)$. Zone I was the projectile-target interaction area, which required a finer mesh than other areas.

The same linear elastic and kinematic plastic hardening material model ( ${ }^{*}$ MAT_PLASTIC_KINEMATIC) was adopted for both the projectile and the deceleration measurement device. The material parameters of the projectile are listed in Table 3. The concrete target was described by the Holmquist-Johnson-Cook (HJC) model ( ${ }^{*}$ MAT_JOHNS ON_HOLMQUIST_CONCRETE). The HJC model is commonly used to describe the dynamic response of brittle material damage when that material is subject to large strains, high strain rates, and high pressures [29]. Table 4 presents the material parameters of the HJC model for the concrete. The parameters are identical to those given in the original reference [29]. The crack modeling of concrete has been investigated extensively [30, 31]. We pay more attention to the target resistance, not the crack propagation. Thus, the concrete crack modeling will not be considered in this work. The contact between the projectile and the deceleration measurement device was simplified as "TIED" contact (*CONTACT_TIED_NODES_TO_SURFACE), and the contacts between the covers of the testing device (including front cover and rear end cover) and housing were also set as the "TIED" contact. In addition, the contact between the projectile and the concrete target was set as "ERODING" contact ( CONTACT_ERODING_SURFACE _TO_SURFACE).

\section{Results and Discussion}

5.1. Deceleration Signal Processing and Validation Based on the Combination of the Modal Analysis and Spectrum Analysis. Figure 9 shows the deceleration-time curve of the prototype projectile. The curve can be divided into two phases: the acceleration phase in the barrel and the deceleration phase during target penetration. The deceleration signal is composed of the rigid-body deceleration, response signal from stress wave loading/unloading inside the projectile during penetration, and measured noise [3]. The rigid-body deceleration signal is a low-frequency signal, while other response signals are high-frequency signals. Thus, to extract the rigid-body deceleration signal, we first determine the filter cutoff frequency. 
TABLE 3: Material parameters for projectile.

\begin{tabular}{lccc}
\hline Material & Parameter & Value & Parameter \\
\hline & Density, $\rho\left(\mathrm{g} / \mathrm{cm}^{3}\right)$ & 7.8 & Tangent modulus, $E_{t}(\mathrm{MPa})$ \\
Projectile & Young's modulus, $E(\mathrm{GPa})$ & 220 & Hardening parameter, $\beta$ \\
& Poisson's ratio, $\mu$ & 0.284 & Failure strain, $f_{s}$ \\
& Yield stress, $\sigma_{s}(\mathrm{MPa})$ & 1800 & \\
\hline
\end{tabular}

TABLE 4: Material parameters for concrete target.

\begin{tabular}{|c|c|c|c|c|}
\hline Material & Parameter & Value & Parameter & Value \\
\hline \multirow{11}{*}{ Concrete } & Density, $\rho\left(\mathrm{g} / \mathrm{cm}^{3}\right)$ & 2.44 & Normalized maximum strength, $S_{\max }$ & 7.0 \\
\hline & Young's modulus, $E$ (GPa) & 20.68 & Crushing pressure, $P_{c}(\mathrm{GPa})$ & 0.016 \\
\hline & Plastic volume modulus, $K(\mathrm{GPa})$ & 14.86 & Crushing volumetric strain, $\mu_{c}$ & 0.001 \\
\hline & Normalized cohesive strength, $A$ & 0.79 & Locking pressure, $P_{l}(\mathrm{GPa})$ & 0.80 \\
\hline & Normalized pressure hardening, $B$ & 1.6 & Locking volumetric strain, $\mu_{l}$ & 0.10 \\
\hline & Strain rate coefficient, $C$ & 0.007 & Damage constant, $D_{1}$ & 0.04 \\
\hline & Pressure hardening exponent, $N$ & 0.61 & Damage constant, $D_{2}$ & 1.0 \\
\hline & Uniaxial compressive strength, $f_{c}(\mathrm{MPa})$ & 48 & Pressure constant, $K_{1}(\mathrm{GPa})$ & 85 \\
\hline & Maximum tensile hydrostatic pressure, $T(\mathrm{GPa})$ & 0.004 & Pressure constant, $K_{2}(\mathrm{GPa})$ & -171 \\
\hline & Plastic strain before fracture, $\varepsilon_{f_{\min }}$ & 0.01 & Pressure constant, $K_{3}(\mathrm{GPa})$ & 208 \\
\hline & Failure strain, $f_{s}(\mathrm{MPa})$ & 0.38 & & \\
\hline
\end{tabular}

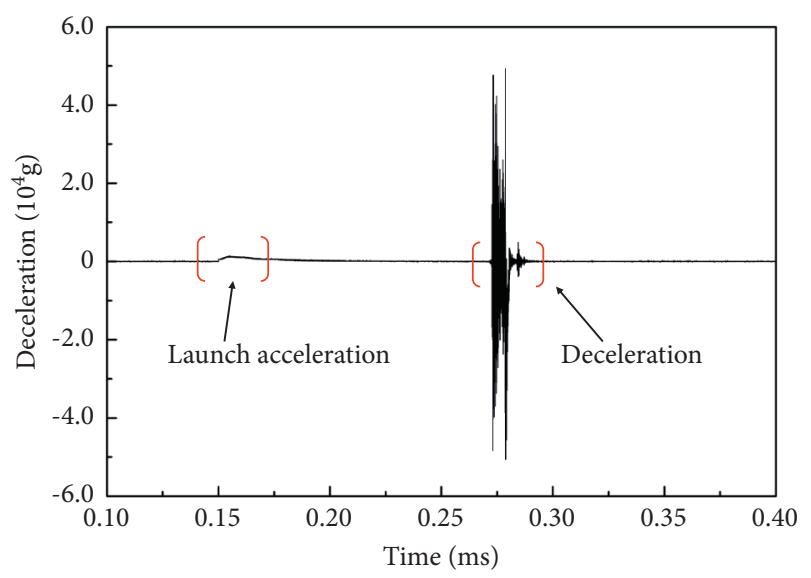

Figure 9: Deceleration-time curve of the prototype projectile.

Numerous researchers have performed studies to determine the filter cutoff frequency of the signal and to effectively extract rigid-body deceleration. Forrestal [32] installed two accelerometers with different projectile positions. During data analysis and processing, the deceleration data of the two test points were gradually filtered with a certain frequency step length from high to low. When the low-pass filter frequency reached $1 \mathrm{kHz}$, there was no difference between the two filtered curves. Therefore, the filtered waveforms were considered as rigid-body deceleration. Some other scholars have evaluated a method to determine the filter cutoff frequency by combining modal analysis and spectrum analysis [33]. Due to its convenience and feasibility from [33], our study adopted this method to determine the filter cutoff frequency.

The finite element modal analysis is performed on the prototype and the scaling model projectile (including the deceleration measurement device), respectively. The first three orders of modal frequency are listed in Table 5. The first column in Table 5 provides the first-order modal frequency of the prototype projectile and the scaling model projectile. Figure 10 shows the spectrum of the deceleration signal after the Fast Fourier Transformation (FFT). From the first-order modal frequency and spectrum of deceleration data, the first wave valley of the spectrum $(3.5 \mathrm{kHz}$ and $2.7 \mathrm{kHz}$ ) was selected as the filter cutoff frequency of the prototype and the scaling model projectile, respectively.

With determined cutoff frequency, we obtained the rigidbody deceleration signal by Butterworth low-pass filtering. The results are shown in Figures 11 and 12 (the red curves). Then, integrations of the deceleration-time data were performed to obtain the striking velocity, which can be compared to the striking velocities measured by the high-speed camera. The blue curves in Figures 11 and 12 show single integrations of deceleration versus time. With these integrations, striking velocities were calculated as $314.8 \mathrm{~m} / \mathrm{s}$ and $317.6 \mathrm{~m} / \mathrm{s}$. Table 6 lists the velocity results obtained using the two methods; $V_{o i}$ represents the velocity obtained by integration, and $V_{o t}$ represents the result captured by the high-speed camera. 
TABLE 5: First three orders of modal frequency of the prototype and scaling model projectile (including the deceleration measurement device).

\begin{tabular}{lccc}
\hline Structure & 1st $(\mathrm{Hz})$ & 2nd $(\mathrm{Hz})$ & $3 \mathrm{rd}(\mathrm{Hz})$ \\
\hline Prototype & 4041.9 & 6952.3 & 8564.2 \\
Scaling model & 3905.0 & 6183.0 & 9239.9 \\
\hline
\end{tabular}

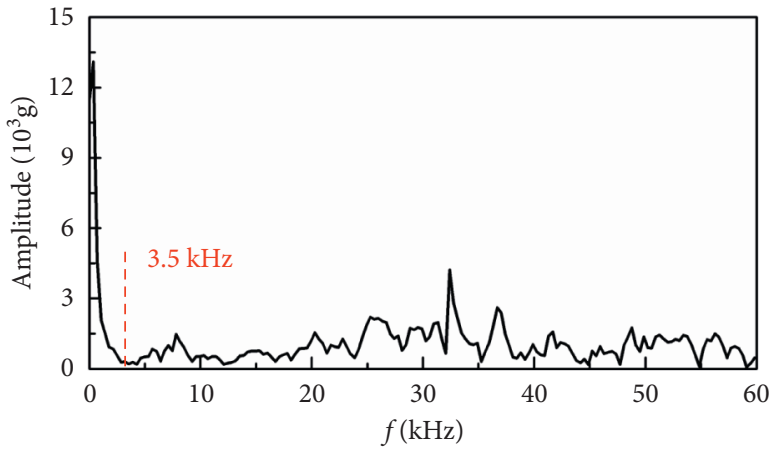

(a)

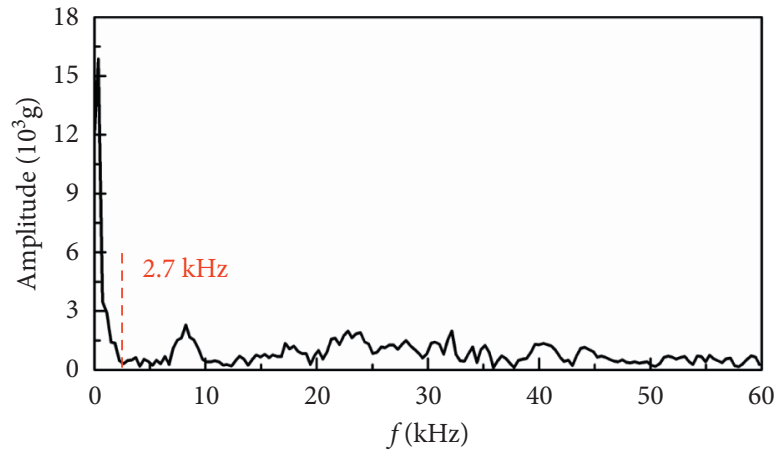

(b)

FIgURE 10: Spectrum of the deceleration-time curve of the (a) prototype and (b) nonproportional scaling model.

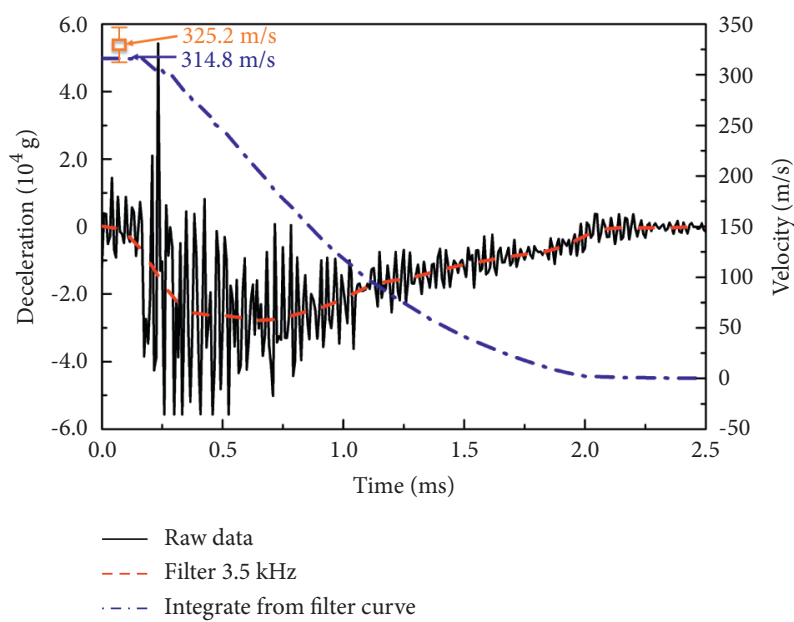

Figure 11: Curve of deceleration and velocity dependence on time for the prototype projectile.

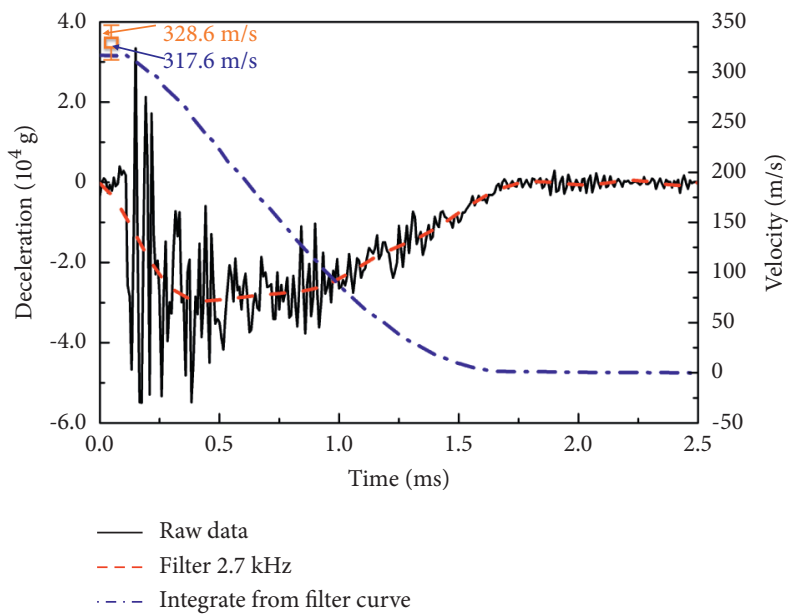

FIGURE 12: Curve of deceleration and velocity dependence on time for the nonproportional scaling projectile. 
TABLE 6: Comparison of the initial striking velocity.

\begin{tabular}{lccr}
\hline Structure & $V_{o i}(\mathrm{~m} / \mathrm{s})$ & $V_{o t}(\mathrm{~m} / \mathrm{s})$ & Deviation $(\%)$ \\
\hline Prototype & 314.8 & 325.2 & 3.20 \\
Model & 317.6 & 328.6 & 3.35 \\
\hline
\end{tabular}

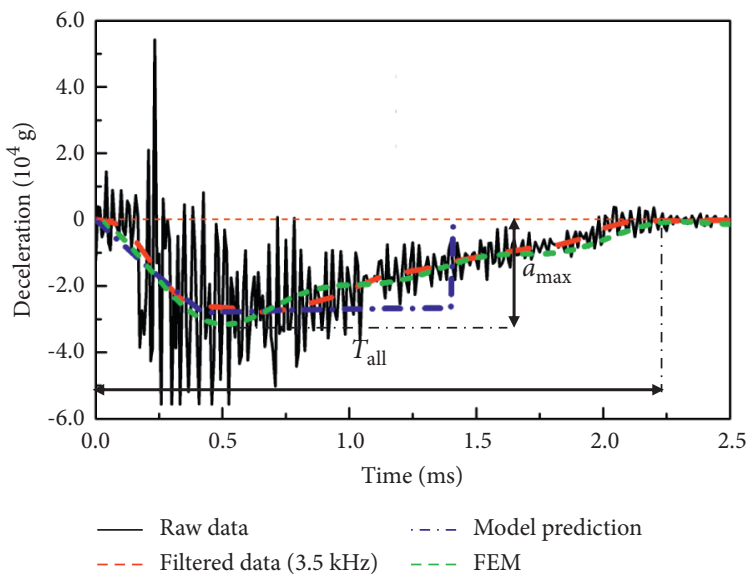

(a)

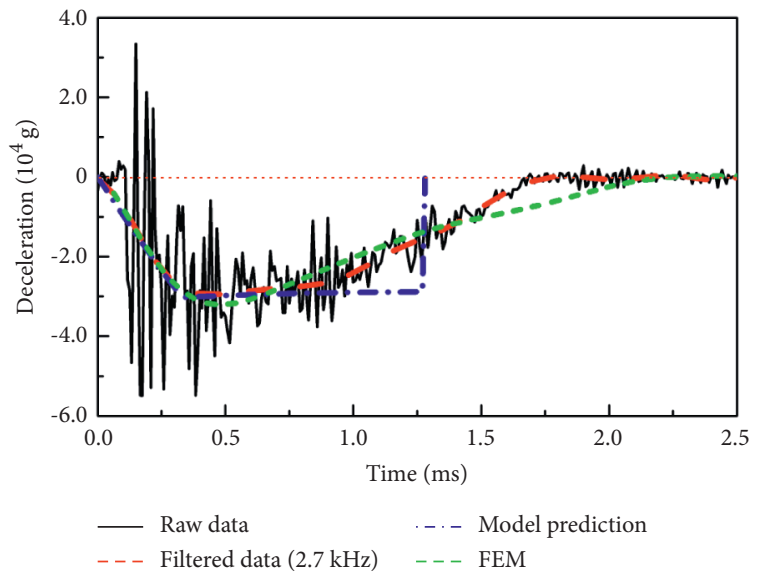

(b)

Figure 13: Comparison of the deceleration-time curves from test, simulation, and empirical model. (a) Prototype projectile; (b) nonproportional model.

Comparing the two methods, the variations are $3.20 \%$ and $3.35 \%$, respectively, which demonstrates the validity and accuracy of the testing deceleration data.

\subsection{Equivalence Validation of Rigid-Body Deceleration for the} Prototype and Nonproportional Scaling Model. Based on the validity and reliability of the test data, the verification of the rigid-body deceleration equivalence of the prototype projectile and scaling projectile was performed in this section. Figure 13(a) shows the comparison of the test, simulation, and empirical model prediction (from equation (4)) of the prototype. All the simulation results in Figure 13 are processed by low-pass filtering, which represents the rigid-body deceleration. The rigid-body deceleration from the test and simulation first increased rapidly, then maintained for a period of time, and finally decreased slowly during the penetration process. The deceleration-time curve from the simulation was in general agreement with that from the test. The rigid-body deceleration can be characterized by the maximum deceleration $\left(a_{\max }\right)$ during penetration and the time to complete penetration $\left(T_{\mathrm{all}}\right)$. As shown in Table 7 , $a_{\max }$ and $T_{\text {all }}$ of rigid-body deceleration from the simulation and test were extracted. The deviations of the $a_{\max }$ and $T_{\text {all }}$ were $11 \%$ and $3.27 \%$, respectively, which indicated the effectiveness of simulation results. In addition, a decelerationtime curve of the empirical model was obtained from equation (4). The maximum deceleration $\left(a_{\max }\right)$ from the model prediction was consistent with the test, and the deviation was $-3.0 \%$. The empirical model cannot accurately describe the process of the slow decrease of the overload peak due to defects, so $T_{\text {all }}$ from the model predicted cannot accurately represent the penetration time. Figure 13(b) shows the comparison of the test, simulation, and model prediction (from equation (4)) of the scaling model. From Figure 13(b), the rigid-body curves all show a rapid increase and then a slow decrease trend. Compared with the test, the errors of $a_{\max }$ and $T_{\text {all }}$ from the simulation are $6.26 \%$ and $11.2 \%$, respectively, and the error of $a_{\max }$ from model prediction is $-0.8 \%$, indicating the reliability of the simulation and model prediction.

Figure 14 shows the comparison of the experimental deceleration-time curves of the prototype and the nonproportional model. The curve patterns are nearly consistent after low-pass filtering, and they all exhibit a similar tendency. The detailed comparison is shown in the first two columns of Table 8. Compared with the prototype projectile, the relative deviations of $a_{\max }$ and $T_{\text {all }}$ from the scaling model were $6.76 \%$ and $-12.1 \%$, respectively. The results indicate that the rigid-body deceleration of the nonproportional model is in general agreement with that of the prototype projectile. This makes it reasonable and feasible to investigate the reliability and survivability of the projectile-borne components for the prototype projectile by using the nonproportional scaling model.

The FEM and empirical model were used to further verify the equivalence of rigid-body deceleration (in Figure 15). From simulation analysis, $a_{\max }$ and $T_{\text {all }}$ of the prototype are in general agreement with the scaling model, and the relative deviations were $2.18 \%$ and $5.4 \%$, respectively (in Table 8). From empirical model prediction, 
TABLE 7: Detailed comparison of rigid-body deceleration for the test, FEM, and model prediction.

\begin{tabular}{lcccccccr}
\hline \multirow{2}{*}{ Structure } & \multicolumn{2}{c}{ Test } & \multicolumn{3}{c}{ FEM } & \multicolumn{3}{c}{ Model prediction } \\
& $a_{\max }(\mathrm{g})$ & $T_{\text {all }}(\mathrm{ms})$ & $a_{\max }(\mathrm{g})$ & $T_{\text {all }}(\mathrm{ms})$ & Error $(\%)$ & $a_{\max }(\mathrm{g})$ & $T_{\text {all }}(\mathrm{ms})$ & Error $(\%)$ \\
\hline Prototype & 27817 & 2.14 & 30882 & 2.21 & $11 / 3.27$ & 26981 & - & -3.0 \\
Mode & 29698 & 1.88 & 31556 & 2.09 & $6.26 / 11.2$ & 29465 & - \\
\hline
\end{tabular}

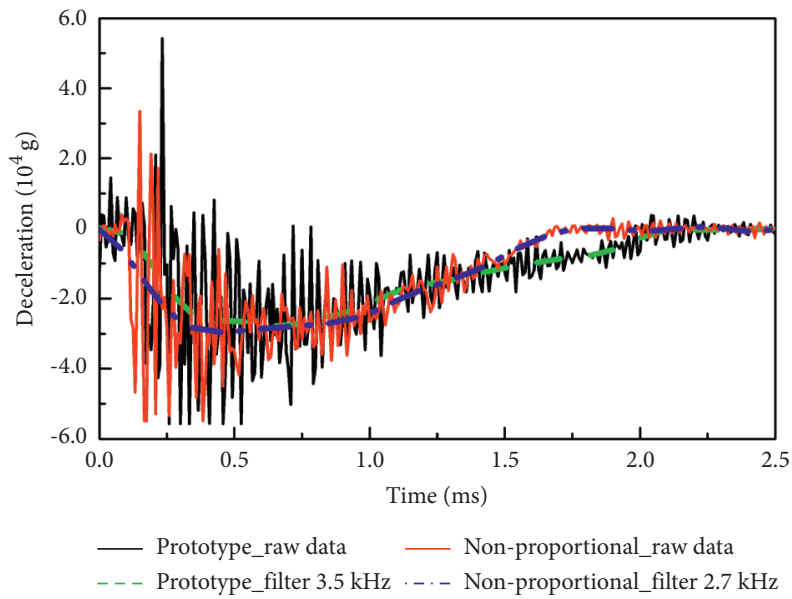

FiguRE 14: Experimental deceleration-time curves of the prototype and nonproportional scaling model.

TABLE 8: Detailed comparison of rigid-body deceleration between the prototype and scaling model.

\begin{tabular}{|c|c|c|c|c|c|c|}
\hline \multirow{2}{*}{ Structure } & \multicolumn{2}{|c|}{ Test } & \multicolumn{2}{|c|}{ FEM } & \multicolumn{2}{|c|}{ Model prediction } \\
\hline & $a_{\max }(\mathrm{g})$ & $T_{\text {all }}(\mathrm{ms})$ & $a_{\max }(\mathrm{g})$ & $T_{\text {all }}(\mathrm{ms})$ & $a_{\max }(\mathrm{g})$ & $T_{\text {all }}(\mathrm{ms})$ \\
\hline Prototype & 27817 & 2.14 & 30882 & 2.21 & 26981 & - \\
\hline Nonproportional & 29698 & 1.88 & 31556 & 2.09 & 29456 & - \\
\hline Deviation (\%) & 6.76 & -12.1 & 2.18 & 5.4 & 9.17 & - \\
\hline
\end{tabular}

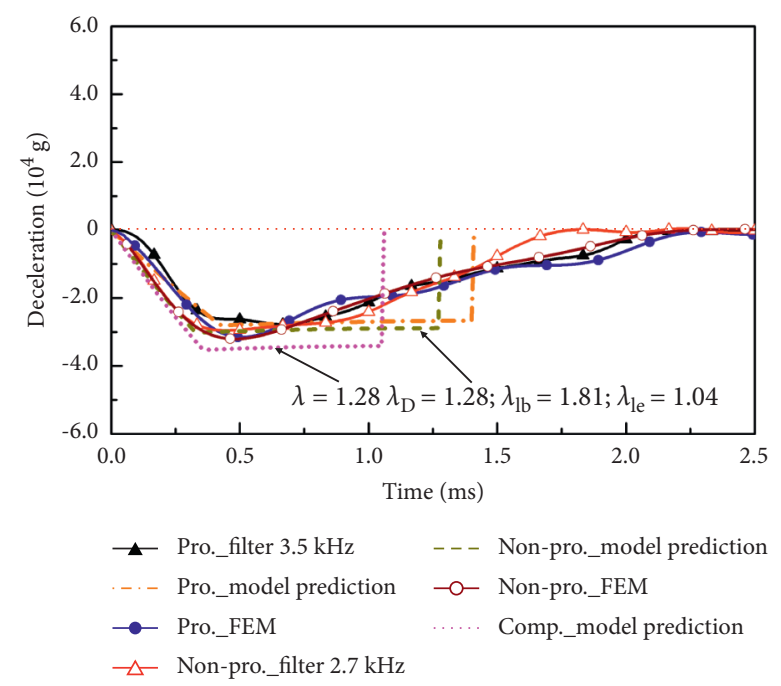

FIGURE 15: Rigid-body deceleration-time curves of the test, FEM, and empirical model for the prototype and nonproportional scaling model. 
the rigid-body deceleration magnitude $\left(a_{\max }\right)$ of the nonproportional model is slightly higher than that of the prototype, while the duration $\left(T_{\text {all }}\right)$ is slightly shorter. The relative deviation of $a_{\max }$ was $9.17 \%$ (in Table 8). The rigidbody deceleration of the complete-similitude model shows significant differences from that of the prototype (in Figure 15). Thus, compared with the complete-similitude model, the rigid-body deceleration from the nonproportional model is basically equivalent with that from the prototype. The scaling model can be used to investigate the reliability and survivability of projectile-borne components inside the prototype projectile.

\section{Conclusion}

In summary, we have proposed a nonproportional scaling model design for penetration test, to investigate the antihigh impact ability of the fuze inside the prototype. This proposed method fulfills the requirements that the complete-similitude scaling scheme is unable to meet, and considers the influence of the mass of the deceleration measurement device. Model predictions, FEM, and penetration tests were used to verify the nonproportional design. The main conclusions are as follows:

(1) A design method of the nonproportional scaling model was proposed. The design criteria of the scaling model projectile considering the mass of the deceleration measurement device were evaluated. The diameter scaling factor and mass scaling factor were adjusted; when $\lambda_{M}=\lambda_{D}^{2}$, the rigid-body deceleration of the scaling model was in agreement with that of the prototype.

(2) In previous research, deceleration signals include rigid-body deceleration, high-frequency response associated with vibrations of the projectile body, and testing noise. We proposed to determine the filter cutoff frequency by combining the spectrum analysis and modal analysis. An improved rigid-body deceleration data was obtained by low-pass filtering the experimental data.

(3) Semi-infinite target penetration tests for both prototype and scaling models were carried out, and deceleration measurement devices of the same dimension were attached for deceleration measurement. The rigid-body deceleration similarity between the prototype and the nonproportional scaling model was verified. Compared with the prototype test, the deviations of the rigid-body deceleration magnitude and duration from the scaling model are $6.76 \%$ and $-12.1 \%$, respectively. This indicates the internal overloading situation of the nonproportional model is in general agreement with that of the prototype projectile.

\section{Abbreviations}

M: Projectile mass in equation (1)

$D$ : Projectile diameter in Figure 2 le: Internal cavity length of the projectile in Figure 2

$l b$ : Shank length of the projectile in Figure 2

$\beta$ : Head volume coefficient of the projectile, which is calculated by caliber radius head $(\mathrm{CRH})$ from reference [22]

$a$ : Penetration rigid-body deceleration

$N$ : Projectile nose factor in equation (5)

$h t$ Wall thickness of the projectile in Figure 2

lh: Head length of the projectile in Figure 2

$\xi$ : Dimensionless wall thickness $\xi=h t / D$ in equation (1)

$\xi_{p}$ : Dimensionless wall thickness of the prototype projectile $\xi_{p}=h t_{p} / D_{p}$

$\xi_{m}$ : Dimensionless wall thickness of the scaling model projectile $\xi_{m}=h t_{m} / D_{m}$

$\lambda_{M}$ : Mass scaling factors

$\lambda_{D}:$ Diameter scaling factors

$\lambda_{l b}$ : Scaling factor of shank length

$\lambda_{l e}$ : Scaling factor of internal cavity length

$\lambda_{a}$ : Scaling factor of penetration deceleration

$\varphi_{m}$ : Dimensionless cavity length of the scaling model $\varphi_{m}=l e_{m} / D_{m}$

$\varphi_{p}:$ Dimensionless cavity length of the prototype projectile $\varphi_{p}=l e_{p} / D_{p}$

$\rho: \quad$ Density of the projectile

$\rho_{t}$ : Density of the target

$\psi$ : Caliber radius head (CRH) in equation (5)

$R$ : Target resistance parameter

$H$ : Penetration depth

$v$ : Penetration instantaneous velocity in equation (4)

$v_{o}$ : Striking velocity of the projectile

$m_{t}$ : Mass of deceleration measurement device (or fuze)

$k$ : Mass ratio of the measurement device to the prototype projectile $k=m_{t} / M_{p}$.

\section{Data Availability}

The data used to support the findings of this study are included within the article.

\section{Conflicts of Interest}

The authors declare that they do not have any commercial or associative interest that represents conflicts of interest in connection with the work submitted.

\section{Acknowledgments}

The authors would like to thank XiaoFeng Li, from the School of Mechatronic Engineering, Beijing Institute of Technology, for his help on the development of the missileborne storage device during the penetration deceleration measurement.

\section{References}

[1] W. Zhang, L. Chen, J. Xiong, and Y. Ma, "Ultra-high g deceleration-time measurement for the penetration into steel target," International Journal of Impact Engineering, vol. 34, no. 3, pp. 436-447, 2007. 
[2] C. Glößner, S. Moser, R. Külls et al., "Projectile penetration testing of granular materials," Experimental Mechanics, vol. 57, no. 2, pp. 261-272, 2017.

[3] Lundgren, "A strain gage based projectile health monitor and salvage indicating circuit for kinetic energy penetrating projectiles," in Proceedings of the 53th NDIA Fuze Conference, Lake Buena Vista, FL, USA, May 2009.

[4] J. Pabio, Analysis of the Deceleration Signal of a Missile during a Hard-Target Attack, Laval University, Quebec, Canada, 1997.

[5] T. L. Warren, M. J. Forrestal, and P. W. Randles, "Evaluation of large amplitude deceleration data from projectile penetration into concrete targets," Experimental Mechanics, vol. 54, no. 2, pp. 241-253, 2014.

[6] D. M. Zhang, S. Q. Gao, S. H. Niu, and H. P. Liu, "Study on collision of threaded connection during impact," International Journal of Impact Engineering, vol. 106, pp. 133-145, 2017.

[7] Y. Guo, Y. Wei, Z. Yang, C. Huang, X. Wu, and Y. Qiuyun, "Nonlinearity of interfaces and force transmission of bolted flange joints under impact loading," International Journal of Impact Engineering, vol. 109, pp. 214-223, 2017.

[8] E. Buckingham, "On physically similar systems: illustrations of the use of dimensional analysis," Physical Review, vol. 4, no. 4, pp. 345-376, 1914.

[9] J. D. Clayton, "Dimensional analysis and extended hydrodynamic theory applied to long-rod penetration of ceramics," Defence Technology, vol. 12, no. 4, pp. 334-342, 2016.

[10] C. E. J. Anderson and J. P. Riegel, “A penetration model for metallic targets based on experimental data," International Journal of Impact Engineering, vol. 80, pp. 24-35, 2015.

[11] Q. M. Li and X. W. Chen, "Dimensionless formulae for penetration depth of concrete target impacted by a non-deformable projectile," International Journal of Impact Engineering, vol. 28, no. 1, pp. 93-116, 2003.

[12] X. W. Chen and Q. M. Li, "Deep penetration of a non-deformable projectile with different geometrical characteristics," International Journal of Impact Engineering, vol. 27, no. 6, pp. 619-637, 2002.

[13] C. G. Chai, A. G. Pi, Q. M. Li, and F. L. Huang, "Similarities in the penetration depth of concrete impacted by rigid projectiles," Acta Mechanica Sinica, vol. 36, pp. 1294-1301, 2020.

[14] Z. J. Song, M. C. Xu, T. Moan, and J. Pan, "Dimensional and similitude analysis of stiffened panels under longitudinal compression considering buckling behaviours," Ocean Engineering, vol. 187, Article ID 106188, 2019.

[15] J. J. Wu, "The complete-similitude scale models for predicting the vibration characteristics of the elastically restrained flat plates subjected to dynamic loads," Journal of Sound and Vibration, vol. 268, no. 5, pp. 1041-1053, 2003.

[16] S. D. Rosa, F. Franco, and T. Polito, "Structural similitudes for the dynamic response of plates and assemblies of plates," Mechanical Systems and Signal Processing, vol. 25, no. 3, pp. 969-980, 2011.

[17] M. Antoniou, N. Nikitas, I. Anastasopoulos, and R. Fuentes, "Scaling laws for shaking table testing of reinforced concrete tunnels accounting for post-cracking lining response," Tunnelling and Underground Space Technology, vol. 101, Article ID 103353, 2020

[18] R. E. Oshiro and M. Alves, "Scaling of cylindrical shells under axial impact," International Journal of Impact Engineering, vol. 34, no. 1, pp. 89-103, 2007.

[19] M. Alves and R. E. Oshiro, "Scaling the impact of a mass on a structure," International Journal of Impact Engineering, vol. 32, no. 7, pp. 1158-1173, 2006.
[20] Z. Luo, Y. P. Zhu, X. Y. Zhao, and D. Y. Wang, "Determining dynamic scaling laws of geometrically distorted scaled models of a cantilever plate," Journal of Engineering Mechanics, vol. 142, no. 4, Article ID 4015108, 2015.

[21] Q. Song, Y. X. Dong, M. Cui, and B. Yu, "A similarity method for predicting the residual velocity and deceleration of projectiles during impact with dissimilar materials," Advances in Mechanical Engineering, vol. 9, no. 7, pp. 1-14, 2017.

[22] A. N. Dancygier, "Scaling of non-proportional non-deforming projectiles impacting reinforced concrete barriers," International Journal of Impact Engineering, vol. 24, no. 1, pp. 33-55, 2000.

[23] M. J. Forrestal, D. J. Frew, J. P. Hickerson, and T. A. Rohwer, "Penetration of concrete targets with deceleration-time measurements," International Journal of Impact Engineering, vol. 28, no. 5, pp. 479-497, 2003.

[24] M. J. Forrestal, B. S. Altman, J. D. Cargile, and S. J. Hanchak, "An empirical equation for penetration depth of ogive-nose projectiles into concrete targets," International Journal of Impact Engineering, vol. 15, no. 4, pp. 395-405, 1994.

[25] M. J. Forrestal, D. J. Frew, S. J. Hanchak, and N. S. Brar, "Penetration of grout and concrete targets with ogive-nose steel projectiles," International Journal of Impact Engineering, vol. 18, no. 5, pp. 465-476, 1996.

[26] D. J. Frew, M. J. Forrestal, and S. J. Hanchak, "Penetration experiments with limestone targets and ogive-nose steel projectiles," Journal of Applied Mechanics, vol. 67, no. 4, pp. 841-845, 1999.

[27] J. B. Men, S. Y. Sui, and J. W. Jiang, "Mesh dependency for numerical simulation of concrete penetration," Transactions of Beijing Institute of Technology, vol. 25, no. 8, pp. 659-662, 2005.

[28] J. A. Zukas and D. R. Scheffler, "Practical aspects of numerical simulations of dynamic events: effects of meshing," International Journal of Impact Engineering, vol. 24, no. 9, pp. 925-945, 2000.

[29] T. J. Holmquest, G. R. Johnson, and W. H. Cook, “A computational constitutive model for concrete subjected to large strains, high strain rates, and high pressures," in Proceedings of the Fourteenth International Symposium on Ballistics, pp. 591-600, Quebec, Canada, September 1993.

[30] T. Rabczuk and T. Belytschko, "A three-dimensional large deformation meshfree method for arbitrary evolving cracks," Computer Methods in Applied Mechanics and Engineering, vol. 196, no. 29-30, pp. 2777-2799, 2007.

[31] T. Rabczuk, G. Zi, S. Bordas, and N. X. Hung, "A simple and robust three-dimensional cracking-particle method without enrichment," Computer Methods in Applied Mechanics and Engineering, vol. 199, no. 37-40, pp. 2437-2455, 2010.

[32] M. J. Forrestal and V. K. Luk, "Penetration into soil targets," International Journal of Impact Engineering, vol. 12, no. 3, pp. 427-444, 1992.

[33] Y. Peng, H. Wu, Q. Fang, and Z. Gong, "Deceleration time of projectile penetration/perforation into a concrete target: experiment and discussions," Advances in Structural Engineering, vol. 22, no. 1, pp. 112-125, 2018. 